\title{
Performance Comparison of OFDM System with Different Modulation Methods
}

\author{
Sakshi Sharma \\ Student \\ JMIT, Radaur \\ ECE Department
}

\author{
Rohan Gupta \\ Assistant Professor \\ JMIT, Radaur \\ ECE Department
}

\begin{abstract}
OFDM is a multicarrier technique for transmission, which divides the available spectrum into many carriers with each one of them being modulated by a data stream of low rate. OFDM is similar to FDMA in the sense that the multiple user access is achieved by further dividing the available bandwidth to multiple channels. These sub channels are then allocated to the users. But in OFDM, the spectrum is used much more efficiently by spacing the channels much close to one another. This is achieved by making all the carriers orthogonal to one another, preventing interference between these carriers. The digital data in serial form to be transmitted is split into different data streams, and each one of them is modulated by a separate carrier in the allotted bandwidth. The modulation done can be any one of the forms of modulation applied to digital data, but the most commonly used are quadrature phase shift keying (QPSK), and quadrature amplitude modulation (QAM). When these modulators are used, their outputs are summed linearly, and the output signal is sent to the channel. In this paper we will analyze the performance of different modulation techniques in AWGN channel. We conclude that a different modulation scheme has different bit error rate.
\end{abstract}

\section{General Terms}

OFDM Transmitter, OFDM Receiver, Performance of Modulation Techniques

\section{Keywords}

OFDM, QAM, QPSK, Bit Error Rate

\section{INTRODUCTION}

Orthogonal frequency division multiplexing (OFDM) is one of the popular methods developed in digital communication scenario and its wide application can be seen in digital television and 4G. OFDM is a technique of encoding digital data using several carrier frequencies. The generation of OFDM involves choosing the spectrum required firstly on the basis of input data and then modulation method to be used is selected. The data to be transmitted is assigned to each one of the carriers partially. On the basis of modulation format, amplitude or phase of the carriers is computed. The requisite band is further transformed back to its signal in time domain using the Inverse Fourier Transform. Now a days, most of the applications use Inverse Fast Fourier Transform. Transformation is done very effectively using the IFFT which gives a simple way to ensure that the carrier signals being produced are orthogonal to one another.

If number of sub-carriers used is $N$, also each of these sub carriers is modulated using $M$ different symbols, and then the OFDM symbol alphabet would consist of $M^{N}$ combined symbols. The low- pass equivalent of OFDM signal is expressed as:

$$
\nu(t)=\sum_{k=0}^{N-1} X_{k} e^{j 2 \pi k t / T}, \quad 0 \leq t<T,
$$

where $\left\{X_{k}\right\}$ represents data symbols and $T$ denotes OFDM symbol time. The spacing between sub carriers is $\frac{1}{T}$ which makes the sub carriers orthogonal to one another during each symbol period and this can be illustrated as:

$$
\begin{aligned}
& \frac{1}{T} \int_{0}^{T}\left(e^{j 2 \pi k_{1} t / T}\right)^{*}\left(e^{j 2 \pi k_{2} t / T}\right) d t \\
= & \frac{1}{T} \int_{0}^{T} e^{j 2 \pi\left(k_{2}-k_{1}\right) t / T} d t=\delta_{k_{1} k_{2}}
\end{aligned}
$$

where $(\cdot)^{*}$ represents the complex conjugate operator and $\delta$ represents Kronecker delta.

When during each interval of a symbol, there is integral number of cycles then the carriers are said to be orthogonal. Hence, there will be a null at the centre frequency in spectrum of each sub carrier. Due to this there will be no interference among the carriers and so the carriers could be placed as close as possible to each other. This removes the requirement of overhead spacing between carriers in FDMA. Since the bandwidth of each one of the carriers in an OFDM signal is narrow, so the resulting symbol rate will be low. Due to this, the tolerance of resultant signal to multipath delay spread will be high.

In the past few years, OFDM systems have been acknowledged because of its capability to combat multipath fading and also the data transmission rate is very high for these systems. The use of adaptive transceivers is one of the possible solutions for improvement of the utilization of capacity of time-varying radio channels. When adaptive modulators are used, the transmitter can use either quadrature amplitude modulation or phase-shift-keying modulation format on the basis of state of the channel. The purpose of the using adaptive modulation is to attain maximum data rate with minimum possible bit error rate. For proper recovery of the data, the modulation type chosen is informed to the receiver.

\section{BASIC OFDM SYSTEM MODEL}

This section deals with the transmitter and receiver section of the OFDM system explained in detail. 


\subsection{OFDM Transmitter}

Figure1 represents the transmitter model of OFDM system. The basic components of a transmitter consist of a binary data source, QAM/QPSK modulator and OFDM baseband modulator.

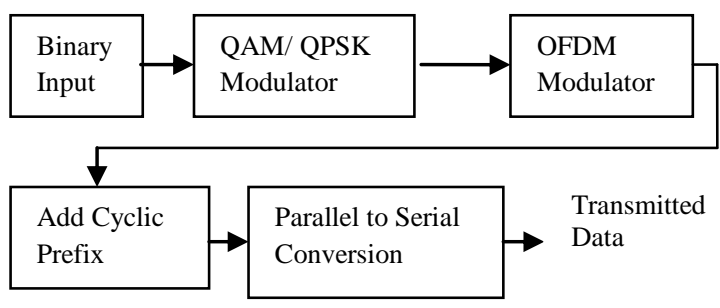

Figure 1 OFDM transmitter

The OFDM signal is the sum of many sub carrier signals which are orthogonal to each other and each of the sub carrier is modulated with the data to be transmitted using either QAM or QPSK. The main RF carrier is further modulated using this composite signal. To convert the symbols to be transmitted into time domain, IFFT is applied to each set.

The binary input data is generated using Bernoulli distribution with the help of Bernoulli Binary Generator block. The amplitude or phase of the signal to be transmitted through channel is changed to modulate it. In the case of OFDM system the amplitude and phase modulation can be used, but frequency modulation cannot be used because subcarriers are orthogonal to each other in frequency and carry independent information. Modulating the carrier frequency will destroy the orthogonality between the subcarriers and this makes frequency modulation unusable for OFDM systems. The OFDM system is modeled with Quadrature Amplitude Modulation and Quadrature Phase Shift Keying modulation schemes. So that best modulation schemes can be found on the analysis of system on the basis of bit error rate. Once modulation is done, an IFFT is carried out because QPSK and QAM symbols are viewed as if they are in frequency domain and hence IFFT block converts them to time domain. The inputs to the IFFT block are $\mathrm{N}$ symbols at a time where $\mathrm{N}$ denotes total subcarriers in the system. Before transmission through the channel, this signal is processed.

Inter symbol interference and inter carrier interference are the main causes of interference in OFDM system. Inter symbol interference refers to the crosstalk between signals separated by signaling interval in the successive frames of same subchannel. On the other hand, inter-carrier interference means crosstalk between adjacent frequency bands of the same FFT frame. This type of interference can be removed by appending a cyclic prefix in the guard interval of an OFDM symbol because of which there will be integer number of cycles in a FFT interval of its delayed versions. The baseband signal to be transmitted is the serial time waveform obtained after addition of the cyclic prefix.

The transmitted signal is then sent over the channel. Various parameters like SNR, multipath etc are controlled by adding a pre decided amount of white noise to the signal that is transmitted. The effect of interference and fading can be studied by modeling the OFDM system with AWGN and multipath fading channels.

\subsection{OFDM Receiver}

Figure2 represents the receiver model of OFDM system. The basic components of a receiver consist of a QAM/QPSK demodulator and OFDM baseband demodulator.

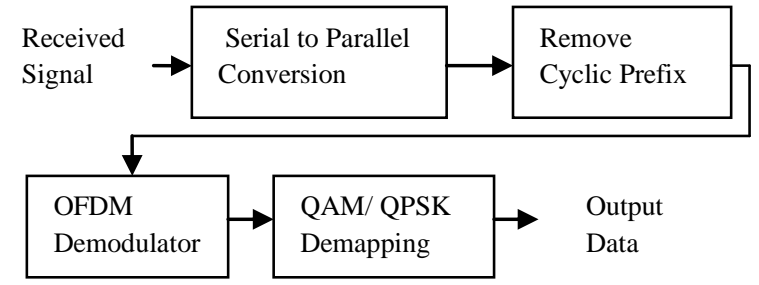

Figure 2 OFDM receiver

Conversion of the received signal back to parallel form from serial form takes place at the receiver. The cyclic prefix is also removed from the data. To get the spectrum of the transmitted signal back, FFT process is applied to the symbols received. The FFT gives the data word which is then combined back to data of the size as same as that was transmitted over the channel. Then this time domain data received from FFT block is given to QAM/QPSK demodulating to get the original data. The performance analysis of this OFDM system is done on the on the basis of bit error rate (BER).

\section{DIFFERENT MODULATION TECHNIQUES}

Modulation can be done by varying the amplitude, phase, or frequency of signal to be transmitted .But in case of an OFDM system, modulation of frequency cannot be done because by doing so orthogonality of sub carriers carrying independent data would be lost. Thus, an OFDM system can be modulated using Quadrature Amplitude Modulation or Quadrature Phase Shift Keying modulation techniques and the best modulation technique can be found on the analysis of bit error rate of the system under different modulation.

\subsection{Quadrature Amplitude Modulation}

In Quadrature Amplitude Modulation (QAM), two amplitude modulated signals can be converted into a single channel by using this method and hence the effective bandwidth can be doubled. In wireless applications QAM is used with pulse amplitude modulation. A QAM signal consists of two quadrature carriers i.e. I and Q signal where one of them is represented by a sine wave and other by a cosine wave. For transmission over the channel, these carriers would be modulated. To retrieve the original information, carriers would be separated at the receiver.

\subsection{Quadrature Phase Shift Keying}

In QPSK, each carrier is differentially encoded and data to be transmitted is mapped into a phase shift format. This requires some initial phase reference and for that a symbol would be appended at the beginning. In QPSK, the phase angles used are $0,90,180$, and 270 degrees. QPSK results in a nonvarying amplitude signal which decreases the problem in amplitude changes due to fading.

\section{PERFORMANCE ANALYSIS OF MODULATION TECHNIQUES}

The performance analysis of OFDM system presented in this section is based on computer simulations. The basic scenario of our simulation is represented by the OFDM transmission 
system performing through multipath fading and AWGN transmission channel.

The Scatter Plot Scope gives us constellation diagram of a modulated signal to tell the modulation characteristics like channel distortions of the signal. The scatter plot also displays the signal's strength at any point in the coverage area. Scatter plot scopes are used to plot the constellation diagram for various modulation schemes in an OFDM system and the results of the simulation are as follows.

The Figure $3-6$ shows the constellation diagrams of OFDM system with 4 - quadrature amplitude modulation , $16-$ QAM, 32 - QAM and quadrature phase shift keying modulation respectively. It is clear that QPSK modulation is better for OFDM system as compared to QAM modulation schemes because in QAM modulation as the modulation index increases, the fading effect increases.

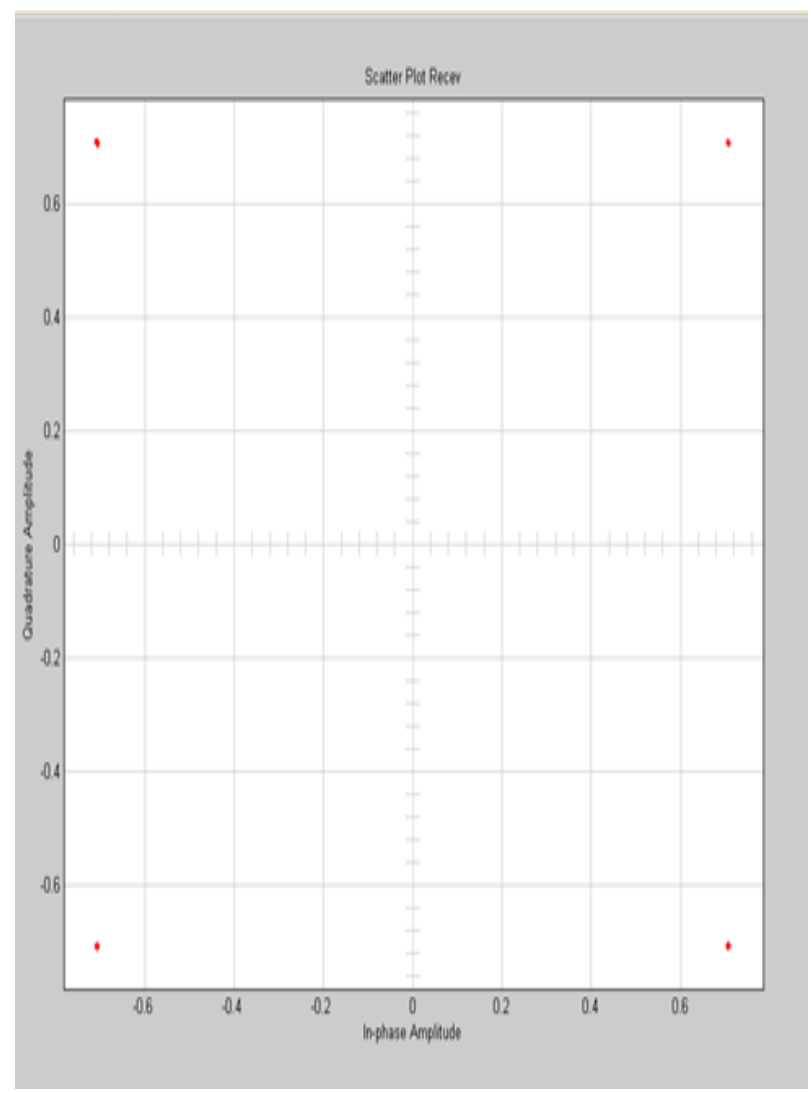

Figure 3 Constellation Diagram of OFDM System with 4 QAM Modulation

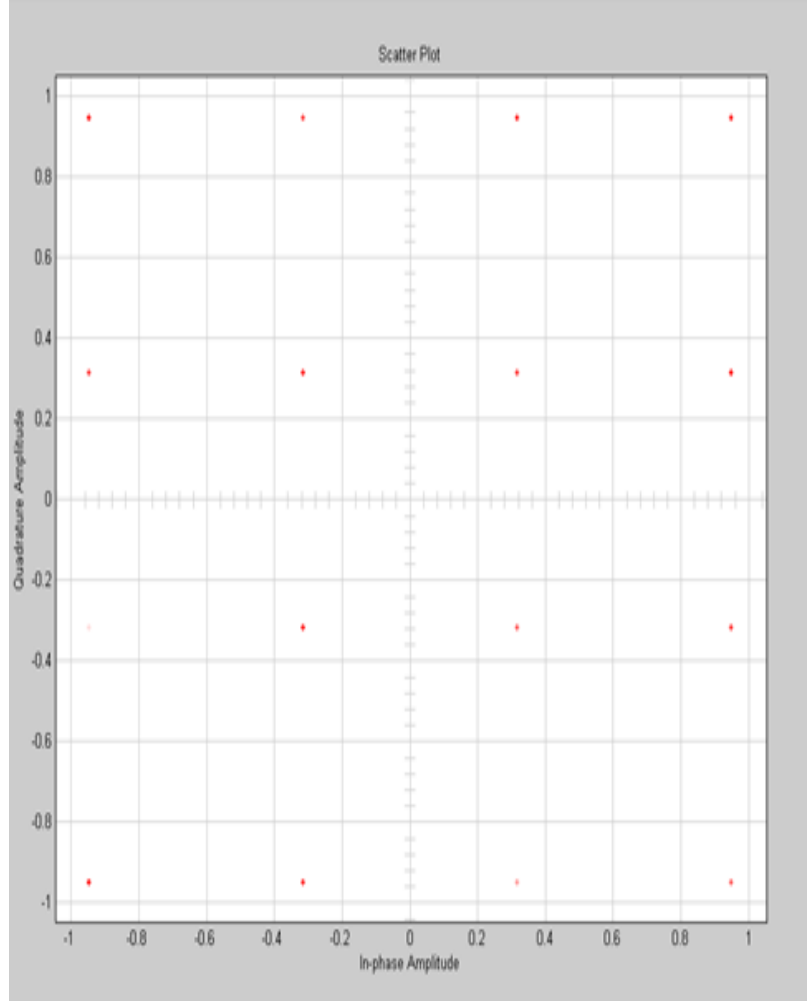

Figure 4 Constellation Diagram of OFDM System with 16 - QAM Modulation

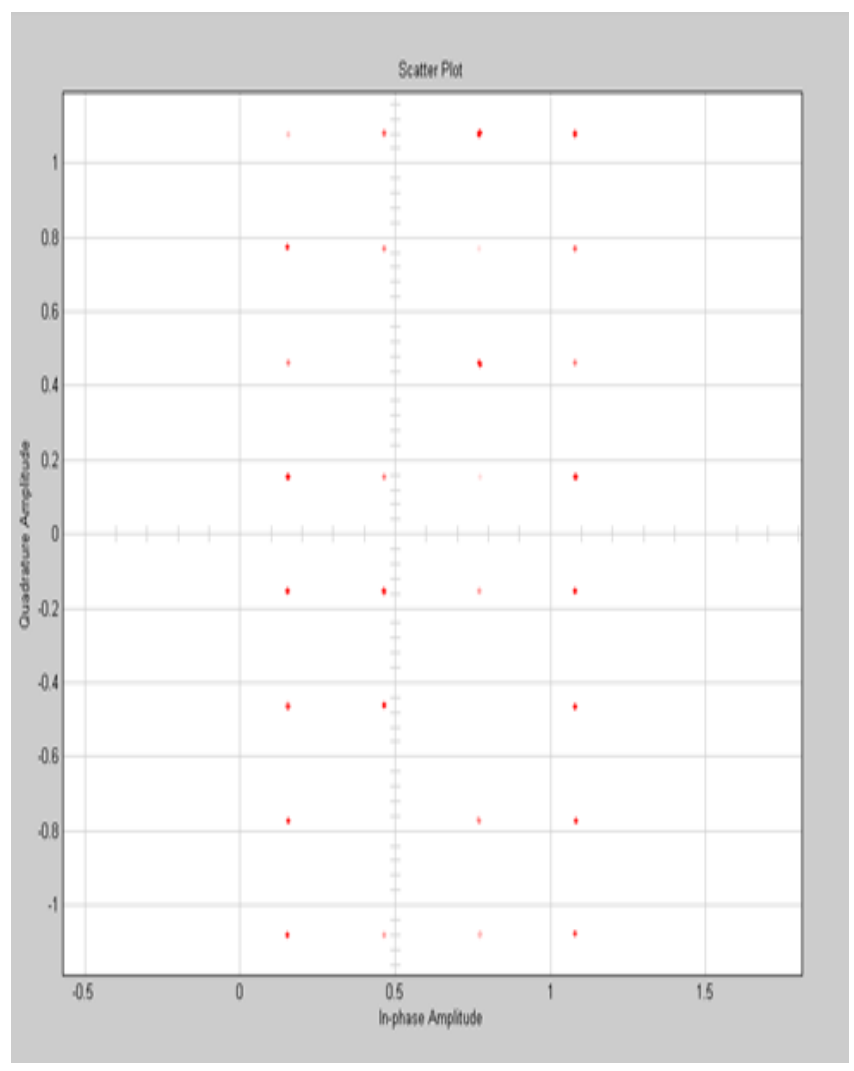

Figure 5 Constellation Diagram of OFDM System with 32 - QAM Modulation 


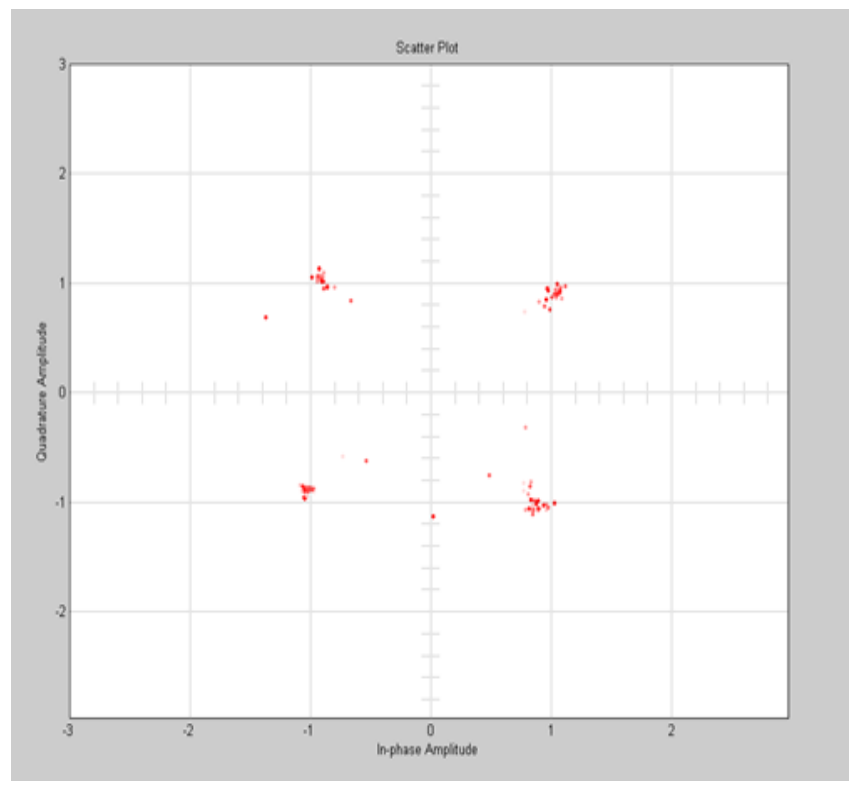

Figure 6 Constellation Diagram of OFDM System with QPSK Modulation

The simulation results of the OFDM system are plotted in term of constellation diagrams. Now the Bit Error Rate of OFDM system is analyzed for different modulation schemes such as 4 - QAM, 16 - QAM, 32 - QAM and QPSK modulation. The BER is calculated with error rate calculation block where the transmitted and received signals are compared.

Table 1 BER of OFDM system with different modulation schemes

\begin{tabular}{|c|l|c|c|c|}
\hline $\begin{array}{c}\text { Sr. } \\
\text { No. }\end{array}$ & $\begin{array}{c}\text { Modulation } \\
\text { Scheme }\end{array}$ & $\begin{array}{c}\text { Total } \\
\text { Number } \\
\text { of Bits }\end{array}$ & $\begin{array}{c}\text { Error } \\
\text { Bits }\end{array}$ & $\begin{array}{c}\text { Bit } \\
\text { Error } \\
\text { rate }\end{array}$ \\
\hline 1. & $4-$ QAM & 191808 & 143902 & 0.7502 \\
\hline 2. & $16-$ QAM & 190656 & 178683 & 0.9372 \\
\hline 3. & $32-$ QAM & 189888 & 183937 & 0.9686 \\
\hline 4. & QPSK & 550000 & 274700 & 0.4996 \\
\hline
\end{tabular}

On comparing these modulation schemes we get that the bit error rate is least for QPSK modulation. Thus from all these systems, QPSK scheme is the best suitable for OFDM system because it has very low bit error rate as compared to other schemes.

\section{CONCLUSION}

Orthogonal Frequency Division Multiplexing (OFDM) is one of the latest technologies to be used for multi carrier digital modulation. A performance comparison of such a system in AWGN and multi path channels is done involving the analysis of the best modulation technique to be used in OFDM system.

OFDM has least BER i.e. $0.4582 \%$ for QPSK scheme and therefore, for accurate transmission OFDM system is the best.

\section{REFERENCES}

[1] Lejiang Guo, Weijiang Wang, Guoshi Wang and Jian Cui, "Research and Implementation of Forest Fire Early Warning System Based on UWB Wireless Sensor Networks", IEEE $2^{\text {nd }}$ International Conference on Communication Systems, Networks and Applications, Vol. 1, pp. 176 - 179, 2010

[2] Namsuk Lee, Yongseouk Choi, Sookjin Lee and Nam Kim, "A New CDMA-Based Bandwidth Request Method for IEEE 802.16 OFDMA/TDD Systems", IEEE 2010 Communications Letters, Vol. 14, pp. $124-126,2010$.

[3] Ciochina C. and Sari H., "A Review of OFDMA and Single-Carrier FDMA", IEEE European Wireless Conference, pp. 706 - 710, 2010.

[4] Xue Li, Ruolin Zhou, Chakravarthy V., Hong S. and Zhiqiang $\mathrm{Wu}$, "Total Intercarrier Interference Cancellation for OFDM Mobile Communication Systems", $7^{\text {th }}$ IEEE Consumer Communications and Networking Conference, pp. 1- 5, 2010.

[5] Wu X.H., Kishk A.A. and Glisson A.W., "MIMOOFDM radar for direction estimation", IET Radar, Sonar \& Navigation, Vol. 4 , Issue 1, pp. 28 - 36, 2010.

[6] Nan Zhou, Xu Zhu, Yi Huang and Hai Lin, "Low complexity cross-layer design with packet dependent scheduling for heterogeneous traffic in multiuser OFDM systems ", IEEE Transactions on Wireless Communications, Vol. 9, Issue 6, pp. 1912 - 1923, 2010.

[7] Sun Pengfei, Morelli Michele and Zhang Li, "Carrier Frequency Offset Tracking in the IEEE 802.16e OFDMA Uplink ", IEEE Transactions on Wireless Communications, Vol. 9, Issue 12, pp. 3613 - 3619, 2010.

[8] Hao Lin, Lele C. and Siohan P., "A pseudo alamouti transceiver design for OFDM/OQAM modulation with cyclic prefix", IEEE $10^{\text {th }}$ Workshop on Signal Processing Advances in Wireless Communications, pp. $300-304$, 2009.

[9] Taha H.J. and Salleh M.F.M., "Performance analysis of QAM-modulation parameters on wavelet packet transform (WPT) and FFT-OFDM system ", IEEE $9^{\text {th }}$ Malaysia International Conference on Communications, pp. $1-5,2009$.

[10] Al-Mawali K.S. and Hussain Z.M., "Performance of bitinterleaved coded OFDM in power line communications with impulsive noise", IEEE International Conference on Advanced Technologies for Communications, pp. 49 53, 2009. 